Chirurg 2014 $\cdot 85: 916$

DOI 10.1007/s00104-014-2888-6

Online publiziert: 17. September 2014

(c) Springer-Verlag Berlin Heidelberg 2014

O. Strobel · M.W. Büchler

Klinik für Allgemein-, Viszeral- und Transplantationschirurgie, Universität Heidelberg

\title{
Fehlgeschlagenes Komplikations- management als Ursache der fallzahlabhängigen Variation der Mortalität nach Leberchirurgie
}

an schweren Komplikationen) im Kontext des Behandlungsvolumens analysiert.

\section{Ergebnisse}

Es wurden 9874 Patienten analysiert, davon 3960 (40,1\%) mit Majorresektionen (mindestens Hemihepatektomie). Die Rate schwerer Komplikationen war $19,6 \%$ in Low-, 19,3\% in Intermediate- und 16,6\% in High-volume-Häusern ( $\mathrm{p}<0,001)$. Die häufigsten schwerwiegenden Komplikationen Lungenversagen $(8,8 \%)$, akutes Nierenversagen $(4,2 \%)$, gastrointestinale Blutung (3,9\%) und Pneumonie (3,1\%) traten in Häusern mit höherem Volumen signifikant weniger häufig auf $(\mathrm{p}<0,05)$. Die typischen chirurgischen Komplikationen nach Leberresektionen (Galleleckage, Hämatom, Leberversagen) wurden nicht analysiert, wahrscheinlich weil sie im NIS nicht getrennt erfasst werden. Eine Infektion im Operationsgebiet trat bei 3,7\% der Fälle auf ohne volumenabhängige Unterschiede. Die Mortalität war 3,7\% in Low-, $3,9 \%$ in Intermediate- und 2,9\% in Highvolume-Häusern $(p<0,001)$. Die wichtigsten unabhängige Risikofaktoren für Mortalität $(\mathrm{p}<0,001)$ waren „low-volume“, ein Alter $\geq 70$ Jahre, hohe Komorbidität, primäre Lebertumoren und Majorresektionen. Die Failure-to-rescue-Rate war in Low- und Intermediate-volume-Häusern mit 16,8 und $16,1 \%$ signifikant höher als in High-volume-Häusern (11,8\%, p=0,032). Nach entsprechender Korrektur für Risikofaktoren hatten Patienten mit einer schwerwiegenden Komplikation in Lowvolume-Häusern ein $40 \%$ erhöhtes Risiko, an der Komplikation zu versterben, im Vergleich zu Patienten in High-volu-
me-Häusern. Über die Zeit konnte die Failure-to-rescue-Rate bei etwa gleichbleibender Komplikationsrate signifikant verbessert werden (Operation in 20002003: $22,0 \%$ vs. Operation in 2008-2011: $12,0 \% ; \mathrm{p}=0,011$ ), sowohl in Low- als auch in High-volume-Häusern.

\section{Diskussion}

Die Studie bestätigt, dass auch in der Leberchirurgie die Ergebnisqualität mit zunehmendem Behandlungsvolumen steigt. Während die Daten zeigen, dass ein Mechanismus für die höhere Mortalität in kleineren Häusern ein weniger erfolgreiches Management der vorwiegend „nichtchirurgischen“ (z. B. pulmonalen) schwerwiegenden Komplikationen ist, lässt die Studie durch die fehlende Erfassung der typischen chirurgisch-technischen Komplikationen keine Aussage über die operative Qualität in Abhängigkeit der Fallzahl zu. Eine gute Ergebnisqualität nach großen chirurgischen Eingriffen ist nur durch gute Chirurgie in Kombination mit einem kompetenten interdisziplinären perioperativen Management möglich.

\section{Korrespondenzadresse}

PD Dr. 0. Strobel

Klinik für Allgemein-, Viszeralund Transplantationschirurgie, Universität Heidelberg,

Im Neuenheimer Feld 110, 69120 Heidelberg

Oliver.Strobel@med.uni-heidelberg.de

Interessenkonflikt. O. Strobel und M.W. Büchler geben an, dass kein Interessenkonflikt besteht. 